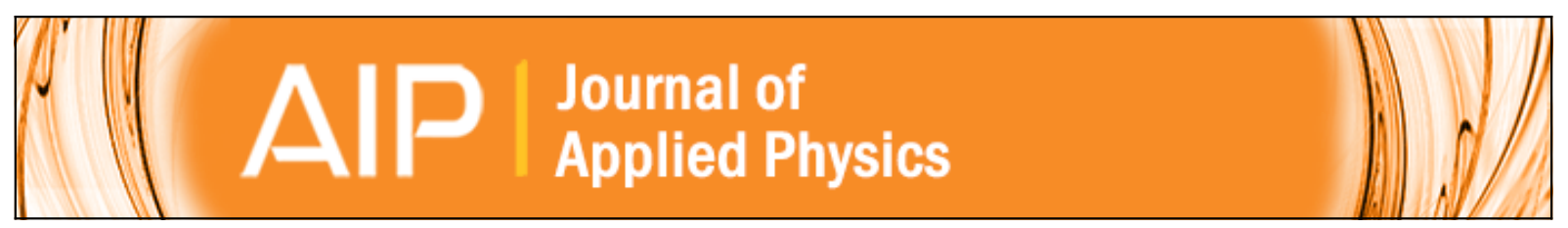

\title{
Model independent $x$-ray standing wave analysis of periodic multilayer structures
}

S. N. Yakunin, I. A. Makhotkin, R. W. E. van de Kruijs, M. A. Chuev, E. M. Pashaev, E. Zoethout, E. Louis, S. Yu.

Seregin, I. A. Subbotin, D. V. Novikov, F. Bijkerk, and M. V. Kovalchuk

Citation: Journal of Applied Physics 115, 134303 (2014); doi: 10.1063/1.4869540

View online: http://dx.doi.org/10.1063/1.4869540

View Table of Contents: http://scitation.aip.org/content/aip/journal/jap/115/13?ver=pdfcov

Published by the AIP Publishing

\section{Articles you may be interested in}

Monte Carlo simulation applied in total reflection x-ray fluorescence: Preliminary results

AIP Conf. Proc. 1529, 66 (2013); 10.1063/1.4804085

A method of measuring gold nanoparticle concentrations by $x$-ray fluorescence for biomedical applications Med. Phys. 40, 051901 (2013); 10.1118/1.4798966

In-situ stoichiometry determination using $x$-ray fluorescence generated by reflection-high-energy-electrondiffraction

J. Appl. Phys. 109, 114305 (2011); 10.1063/1.3592219

Multiplexed biomarker detection using x-ray fluorescence of composition-encoded nanoparticles Appl. Phys. Lett. 97, 263704 (2010); 10.1063/1.3533817

Analysis of octadecyltrichlorosilane treatment of organic thin-film transistors using soft x-ray fluorescence spectroscopy

Appl. Phys. Lett. 86, 232103 (2005); 10.1063/1.1944900

\section{A|P| $\left.\right|_{\text {Applied Physics }} ^{\text {Journal of }}$}

Journal of Applied Physics is pleased to announce André Anders as its new Editor-in-Chief 


\title{
Model independent $x$-ray standing wave analysis of periodic multilayer structures
}

\author{
S. N. Yakunin, ${ }^{1}$ I. A. Makhotkin, ${ }^{2}$ R. W. E. van de Kruijs, ${ }^{3}$ M. A. Chuev, ${ }^{4}$ E. M. Pashaev,${ }^{1}$ \\ E. Zoethout, ${ }^{3}$ E. Louis, ${ }^{2,3}$ S. Yu. Seregin, ${ }^{5}$ I. A. Subbotin, ${ }^{1}$ D. V. Novikov, ${ }^{6}$ F. Bijkerk, ${ }^{3,2}$ \\ and M. V. Kovalchuk ${ }^{1,5}$ \\ ${ }^{1}$ NRC Kurchatov Institute, Moscow, Russia \\ ${ }^{2}$ MESA + Institute for Nanotechnology, University of Twente, Enschede, The Netherlands \\ ${ }^{3}$ FOM Institute DIFFER, Nieuwegein, The Netherlands \\ ${ }^{4}$ IPT RAS, Moscow, Russia \\ ${ }^{5}$ IC RAS, Moscow, Russia \\ ${ }^{6}$ DESY, Hamburg, Germany
}

(Received 6 December 2013; accepted 14 March 2014; published online 1 April 2014)

\begin{abstract}
We present a model independent approach for the analysis of X-ray fluorescence yield modulated by an X-ray standing wave (XSW), that allow a fast reconstruction of the atomic distribution function inside a sample without fitting procedure. The approach is based on the direct regularized solution of the system of linear equations that characterizes the fluorescence yield. The suggested technique was optimized for, but not limited to, the analysis of periodic layered structures where the XSW is formed under Bragg conditions. The developed approach was applied to the reconstruction of the atomic distribution function for $L a N / B N$ multilayers with 50 periods of $43 \AA$ thick layers. The object is especially difficult to analyze with traditional methods, as the estimated thickness of the interface region between the constituent materials is comparable to the individual layer thicknesses. However, using the suggested technique, it was possible to reconstruct width of the $L a$ atomic distribution showing that the $L a$ atoms stay localized within the $L a N$ layers and interfaces and do not diffuse into the $B N$ layer. The analysis of the reconstructed profiles showed that the positions of the center of the atomic distribution function can be estimated with an accuracy of $1 \AA$ A. C 2014 AIP Publishing LLC. [http://dx.doi.org/10.1063/1.4869540]
\end{abstract}

\section{INTRODUCTION}

The X-ray standing wave (XSW) technique ${ }^{1,2}$ is applied to non-destructively reconstruct the atomic profiles in crystals and in periodic ${ }^{3-7}$ or aperiodic stratified structures. ${ }^{3,4,8-10}$ The technique is based on the measurement and analysis of the characteristic signal from specific atoms excited by the XSW formed inside a structure. The position of nodes and antinodes of the XSW formed at Bragg reflection conditions in a periodic layered structure or at total external reflection conditions in a non-periodic structure can be modified by changing the incidence angle. The angular dependent intensity of secondary emission yield from the atoms excited by the XSW is now determined by the overlap between the atomic profile and the electromagnetic field. Knowing the electromagnetic field distribution inside the structure, the atomic distribution can be reconstructed from the measured angular dependent fluorescence yield.

In this paper, we consider the XSW analysis using X-ray fluorescence. The most reliable approach to the atomic profile reconstruction is a simultaneous fit of grazing incidence X-ray reflectivity (GIXR) and XSW data having the atomic profile as a fit parameter. However, this technique is time consuming because of the large amount of fit parameters, and moreover, the outcome may be dependent on the initial model. The complicated data analysis is generally the limiting factor for the application of the XSW technique.

Recently, a model independent approach to the reconstruction of the atomic distribution profile from XSW data was suggested by Cheng et al. ${ }^{11}$ and later extended by Kohli et al. ${ }^{12}$ The work $^{11}$ presents the Fourier transformation of measured fluorescence yields excited by the Bragg-XSW in a single crystalline sample. The Fourier transformation requires the measurement of the angular dependent fluorescence yield at different Bragg reflection orders and therefore requires a highly ordered structure. An extension of the this approach for the analysis of thin film structures with long period XSW was presented by Kohli et al. ${ }^{12}$ This method relies on the XSW data with a large number of fluorescence yield oscillations, and imposes strict requirements on the design of the sample to be analyzed. In the current paper, we present a new approach for a model independent analysis of the XSW data that is based on the direct solution of the ill-defined system of linear equations describing the angular dependent fluorescence yield using the Tichonov regularization technique. ${ }^{13}$ Similarly to Kohli et al., ${ }^{12}$ the presented analysis requires the knowledge of the electromagnetic (EM) field that can be obtained from the analysis of grazing incidence X-ray reflectivity data. ${ }^{14}$

In this paper, we will use XSW to analyze the atomic concentration profiles of $L a$ and pollutant $(K r)$ atoms in short period $L a N / B N$ multilayer mirrors. Such multilayers are considered as very promising reflective optical coatings for next generation EUV lithography ${ }^{15,16}$ at $6.7 \mathrm{~nm}$ wavelength and their optical performance is intrinsically linked to the indepth atomic profiles. The preliminary structural analysis of $L a / B$-based multilayer stacks $\left(L a / B\right.$ and $\left.L a / B_{4} C\right)$ shows high 
interface imperfections because of the intermixing between the $L a$ and $B$ layers. ${ }^{15}$ The passivation of $L a$ with $N$ ions improves the quality of the multilayer mirror. ${ }^{17}$ It can be expected that passivation of both layers has the potential to create diffusion free multilayers because of the chemical stability of $L a N$ and $B N$.

\section{MODELING}

\section{A. XSW data analysis}

The modulation of the intensity of the fluorescence yield from atoms in a film is determined by the electromagnetic field $|E(\theta, z)|^{2}$, depending on the incidence angle $\theta$ and distance $z$ from the film surface, and the atomic distribution function (ADF) $\mathbf{P}$ in the film. For calculations of the EM field, it is necessary to divide the entire film into very thin sub-layers where the thickness of individual sub-layers is much smaller than the thickness of the layers, with each sublayer having a constant atomic concentration. In the dipole approximation, the angular dependence of the fluorescence yield intensity $Y(\theta)$ is calculated as the sum over all sublayer of the products of the electromagnetic field distributions $\left|E\left(\theta, z_{j}\right)\right|^{2}$, and the concentration of fluorescent atoms in each sub-layer $P_{j}$, corrected for the geometrical factor $G(\theta)$ and absorption of the fluorescence radiation

$$
Y(\theta)=G(\theta) \sum_{j} P_{j}\left|E\left(\theta, z_{j}\right)\right|^{2} e^{-\mu_{f} z_{j}} .
$$

Here, $\mu_{f}$ is the averaged linear absorption coefficient at the fluorescence wavelength on the exit path from the film. The geometrical factor takes into account the variation of the beam footprint with the change of the incidence angle. The values of fluorescence yield function $Y(\theta)$ in Eq. (1) are normalized on a total amount of fluorescent photons, in order to account for the cross-section of the X-ray fluorescence process. The ADF $\mathbf{P}$ represents the shape of atomic profile, or mathematically the probability density of a distribution of fluorescent atoms.

If the studied sample is a periodic multilayer structure that contains $N$ bi-layers with thickness $\Lambda$, the ADF $\mathbf{P}$ will have $N$ identical periods. Assuming a perfect periodicity, the multilayer can be presented as one "effective" period where the electromagnetic field distribution is the summed EM field from all periods in the multilayer. The effective EM field that excites fluorescence for the whole multilayer can now be represented as

$$
\bar{I}_{j}(\theta)=G(\theta) \sum_{k=1}^{N}\left|E\left(\theta, z_{j k}\right)\right|^{2} e^{-\mu_{f} z_{j k}},
$$

where $z_{j k}=\Lambda[k-1+(j-1 / 2) / m], j=[1 \ldots m]$ is the number of the sub-layer within one period, $m$ is the number of sublayers in one period, and $k=[1 \ldots N]$ is the number of the period in the multilayer. Formula (1) can then be simplified to

$$
Y\left(\theta_{i}\right)=\sum_{i=1}^{n} \sum_{j=1}^{m} P_{j}^{\prime} \bar{I}_{j}\left(\theta_{i}\right) .
$$

In Eq. (3), $P^{\prime}$ is the ADF along a period. For brevity, the apostrophe will further be omitted.

Having measured the fluorescence angular dependency $Y_{\text {exp }}$, according to the method of least squares the unknown function $P_{j}$ is found by minimizing the function

$$
\chi^{2}=\frac{1}{n-m} \sum_{i} \frac{1}{\sigma_{i}^{2}}\left(Y_{\exp }\left(\theta_{i}\right)-\sum_{j} P_{j} \bar{I}_{j}\left(\theta_{i}\right)\right)^{2},
$$

where $n$ is the number of measured angular points and $\sigma_{i}$ the statistical error of the fluorescence yield measurements.

Generally, $\chi^{2}$ can be minimized using a fit procedure if there is no algebraic solution possible. However, in the case presented here, the problem can be presented as a system of linear equations

$$
\partial \chi^{2} / \partial P_{j}=0
$$

After taking the derivative of $\chi^{2}$, Eq. (5) is transformed into $^{18}$

$$
\hat{A} \mathbf{P}=\mathbf{b},
$$

where

$$
\begin{gathered}
\hat{A}_{j l}=\sum_{i=1}^{n} \frac{\bar{I}_{j}\left(\theta_{i}\right) \bar{I}_{l}\left(\theta_{i}\right)}{\sigma_{i}^{2}}, \\
b_{l}=\sum_{i=1}^{n} \frac{Y_{\exp }\left(\theta_{i}\right) \bar{I}_{l}\left(\theta_{i}\right)}{\sigma_{i}^{2}},
\end{gathered}
$$

and $j, l=[1 \ldots m]$, the number of the sublayer in a multilayer period.

The system of equations (6) can be solved numerically for $\mathbf{P}$. However, due to systematic and statistical experimental errors the reconstructed atomic distribution function may exhibit non-physical features such as negative values and strong fluctuations. This effect is the consequence of an illposed problem. ${ }^{13}$ In order to obtain reasonable solutions, a regularization is introduced in the solving algorithm. The limitation can, e.g., be the smoothness of distribution function. Mathematically this requirement can be introduced by adding the auxiliary term $u$ in the function $\chi^{2}$. We use the following auxiliary term:

$$
\begin{aligned}
u= & \lambda\left[\sum_{j=2}^{m-1}\left(2 P_{j}-P_{j-1}-P_{j+1}\right)^{2}+\cdots\right. \\
& +\left(2 P_{1}-P_{m}-P_{2}\right)^{2}+\cdots \\
& \left.+\left(2 P_{m}-P_{m-1}-P_{1}\right)^{2}\right]
\end{aligned}
$$

wherein the first term defines the smoothness of the profile amplitude within a period. The second and third terms define continuity on the upper and lower interface, respectively. The smoothening coefficient $\lambda$ determines the maximum "allowed" changes from $P_{j}$ to $P_{j+1}$ and should be selected for each separate case individually. The system of equations (6) is then transformed into the system of linear equations 


$$
(\hat{A}+\lambda \hat{D}) \mathbf{P}=\mathbf{b} .
$$

Here, $\hat{D}$ is the regularization Gram matrix

$$
\hat{D}=\left(\begin{array}{ccccc}
2 & -1 & 0 & \cdots & -1 \\
-1 & 2 & -1 & & 0 \\
\vdots & \ddots & \ddots & \ddots & \vdots \\
0 & & -1 & 2 & -1 \\
-1 & \cdots & 0 & -1 & 2
\end{array}\right)
$$

The used Gram matrix is adapted for the periodical structure of multilayer mirrors. A similar type of regularization, although for non-periodic structures, has been successfully applied for the similar problem of reconstructing scatter density profiles from X-ray reflectometry. ${ }^{19}$

The methods and procedures described here will be applied in the experimental section of the paper. Using the described algorithms the XSW data can be analyzed without any pre-assumptions about the atomic distribution.

\section{B. Calculation of the EM field}

The electron density profile (EDP) of the periodic multilayer structure was reconstructed by iterative fitting of model-based reflectivity calculations to the measured GIXR data. In the simplest case, a layer in a multilayer film can be described with 4 parameters: layer thickness, interface thickness, layer density, and a material composition. The changes in the EDP at the interfaces are described by dividing the interface region in equal sub-layers with a thickness of less than $1 \AA$ and assuming a linear transition in the electron density between neighbouring layers. In this model, each interface will still be described with one parameter: the thickness of the region of linear transition. A more complex parameterized description of the EDP in the interface transition regions could be used, but goes beyond the scope of this work. This description of interfaces, as presented here, is preferred over the standard Debye Waller or Nevot-Croce approaches when the calculation of the EM fields in the interface regions is required.

The reflectivity calculations were performed using the Abeles matrix formalism ${ }^{20}$ which requires the multiplication of the characteristic matrices that describe electromagnetic wave penetration through all the layers in the sample, including the sub-layers in the interface regions. If the multilayer has good periodicity and periods can be assumed identical, the Chebishev polynomials can be used ${ }^{21}$ to calculate analytically the $\mathrm{N}^{\text {th }}$ power of the characteristic matrix for each period in the multilayer. This limits the number of matrix multiplications to the calculation of the characteristic matrix for one period only. This approach can be applied if the errors introduced in the parameter determination by aperiodicity of the multilayer are within the general uncertainty of parameter determination.

\section{EXPERIMENTAL}

The GIXR data were measured using a PANalytical X'Pert PRO diffractometer using $C u K_{\alpha 1}$ radiation and a
$4 \times G e(220)$ asymmetrically cut monochromator. The measurements were done using a constant $2 \theta$ step of $0.005^{\circ}$ and 2 $\mathrm{s}$ of exposure at each step. The XSW measurements were performed at the Hasylab E2 beamline of the DESY synchrotron radiation facility. The bending magnet radiation was monochromized for the wavelength of $0.71 \AA$. The fluorescence spectra were measured for $5 \mathrm{~s}$ per angular step using a Roentec energy dispersive detector. Angular scans were repeated in a fixed range of angles around the first Bragg reflection peak, and the signals were summed until the statistical error of the accumulated integral fluorescence yield was better than $1 \%$.

The 50 period $L a N / B N$ multilayer structure was deposited using DC magnetron sputtering of $L a$ and $B_{4} C$ targets using $K r$ as a sputter gas. This number of period was selected as a compromise between the number of periods required for the formation of the contrast X-ray standing wave, the excitation of sufficiently intense fluorescence radiation and the stability of the deposition process. The passivation of $L a$ and $B_{4} C$ layers was done using low energy $N$-ion treatment of the deposited layers. ${ }^{22}$ XPS measurements were used to optimize the parameters of the deposition of $L a N$ and $B N$ films (optimization is not presented here). The ratio of $L a$ layer to period thickness was selected to be 0.4 for optimal optical performance.

\section{RESULTS}

\section{A. Electron density profile reconstruction}

The electron density profile was reconstructed from the fit of the GIXR data in order to calculate the EM field for XSW analysis. The analysis of GIXR data was performed in two steps: first the fit was performed with a model that describes a 50 times repeated structure, the multilayer period, varying the period structure parameters (layer and interface thicknesses and densities), and assuming no period variation along the stack. Separately, a second fit was performed where the parameters of all periods were varied individually. From the first fit, the average period structural parameters and their statistical errors were obtained. From the second fit, the individual parameters of all layers were obtained and their deviation from the average was determined. Comparing both the averaged and individual parameter sets allows checking the applicability of the model with identical periods for the XSW analysis.

The average values were obtained for the model containing 49 identical periods. The parameters of the top layers were fitted separately assuming that contact with ambient gases possibly changes the top layer structure. The best model representation of the EDP is shown in Fig. 1(a). As depicted in Fig. 1(a), each interface consists of two linear segments with different slopes, indicating that one simple linear transition is not good enough. For each parameter, the fit errors were estimated. The parameter values and their errors are shown in Table I in the column "Average."

In the second fit we allowed all 50 periods to have individual layer parameter. This "individual layers" fit varies from the fit with "identical period" mostly with the better match of the Kiessig fringes (Fig. 2). Analyzing the 

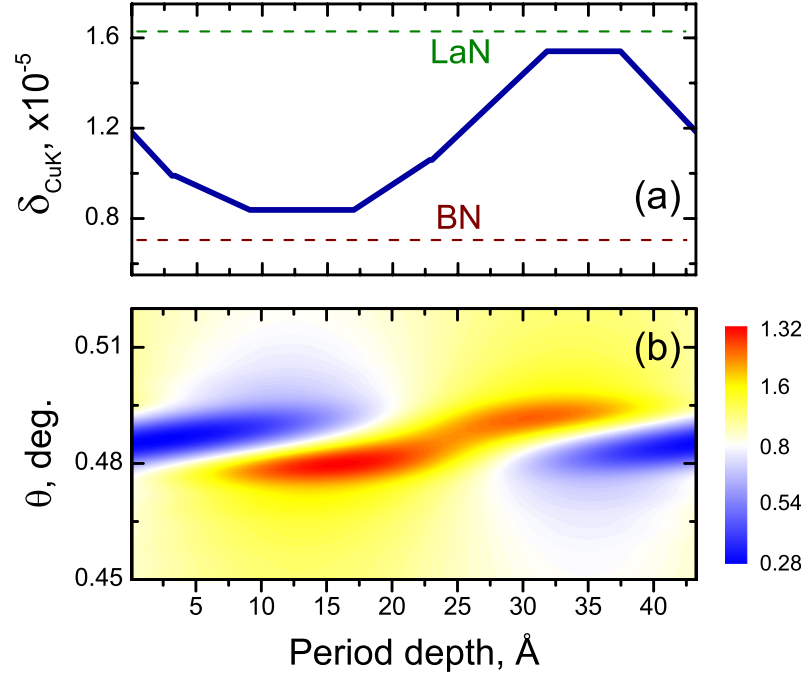

FIG. 1. Reconstructed optical constant profile in the $L a N / B N$ multilayer (a) and EM field distribution in the vicinity of the 1-st Bragg peak (b) calculated for the wavelength of $0.71 \AA$ used in the fluorescence yield measurements.

distribution of individual layer parameters, their standard deviation from their average values is determined and presented in Table I in the column "Deviation." The obtained period thickness of the multilayer determined from both fits was $\Lambda=43.4 \AA$ with a standard deviation of the many-period fit $\mathrm{s}_{\mathrm{D}}=0.24 \AA$, close to the error in the period determination from the fit of the model with identical periods and small compared to the actual value. This confirms that the model with identical periods describes the EDP in the full stack well and may be used for XSW data analysis. The EM field was finally calculated using the Abeles matrix formalism for the reconstructed averaged multilayer profile and summed along the $z$ direction over all periods according to Eq. (2). Figure 1(b) shows the averaged EM field visualizing positions of the nodes and antinodes of the XSW within a period.

From the GIXR EDP, it is observed that approximately $70 \%$ of the period thickness is in the interface state (referring to the two gradients in between the $L a N$ and $B N$ ). This would suggest that there is significant intermixing in the multilayer.

\section{B. Atomic distribution reconstruction}

A typical measured fluorescence spectrum is presented in Fig. 3. Additional to the expected $L a L$ fluorescence yield, fluorescence from $\mathrm{Si}$ (substrate) and $K r$ was detected. $K r$ was used as the magnetron sputtering gas, and its presence indicates trapping of $\mathrm{Kr}$ in the multilayer. The small doublet at

TABLE I. LaN/BN model parameters reconstructed from GIXR. For each parameter we show averages over all the periods and a standard deviation of the values for all 50 periods.

\begin{tabular}{|c|c|c|c|c|c|c|}
\hline & \multicolumn{2}{|c|}{$\begin{array}{c}\text { Layer } \\
\text { thickness, } \AA\end{array}$} & \multicolumn{2}{|c|}{$\begin{array}{l}\text { Top interface transition } \\
\text { layer thickness, } \AA\end{array}$} & \multicolumn{2}{|c|}{$\begin{array}{l}\text { Density, } \\
\mathrm{g} / \mathrm{cm}^{3}\end{array}$} \\
\hline & Average & Deviation & Average & Deviation & Average & Deviation \\
\hline$B N$ & $7.95 \pm 0.16$ & 0.19 & $14.9 \pm 0.2$ & 0.2 & $2.7 \pm 0.05$ & 0.1 \\
\hline LaN & $5.63 \pm 0.08$ & 0.21 & $14.8 \pm 0.2$ & 0.3 & $5.8 \pm 0.1$ & 0.13 \\
\hline
\end{tabular}

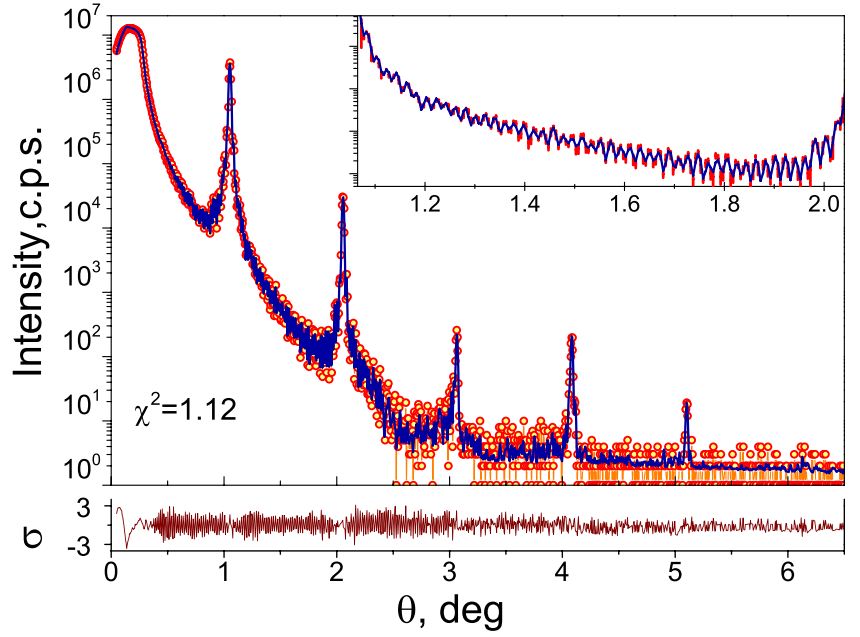

FIG. 2. Experimental and simulated GIXR data for the model with individually defined periods. The insert shows the measured and simulated Kiessig fringes between first and second order Bragg peaks.

$3 \mathrm{keV}$ originates from the $\operatorname{Ar} K_{\alpha}$ and $K_{\beta}$ lines. The intensity of this signal corresponds to the intensity of $A r$ from the ambient environment. For XSW analysis, the background corrected integral intensity of $L a L$ and $K r K$ fluorescence radiation were determined at a range of angles of incidence around the first order Bragg reflection.

The angular dependencies of the $K r$ and $L a$ fluorescence yields calculated based on the direct solution of the system of linear equations (6), using EM fields reconstructed from the GIXR measurements, are presented in Fig. 4. The corresponding reconstructed $L a$ and $K r$ atomic distribution functions are presented in Figures 5(a) and 5(b). Note that all atomic distribution functions presented in Fig. 5 are normalized such that the integral of the function is unity, corresponding to the probability density of the atom distribution in the period of the multilayer structure. Although a very good agreement between simulations and experiments is observed in Fig. 4, the reconstructed atomic distribution functions are clearly non physical when no limitations are introduced in the function, as can be observed from the negative probabilities in Figs. 5(a) and 5(b). Note that even for a non physical solution, the fit goodness for $L a\left(\chi^{2}=2.97\right)$ and for $K r\left(\chi^{2}=3.29\right)$ are not equal to unity. For solutions, that will be discussed further in the article, we will not present the calculated curves but will indicate the values for the

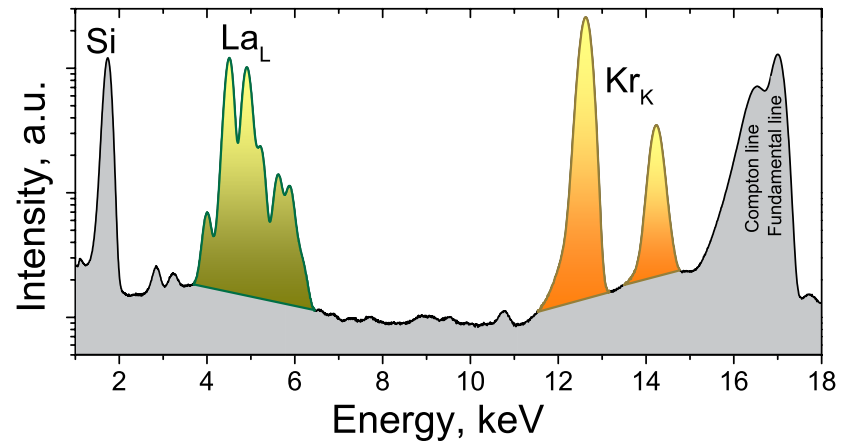

FIG. 3. Typical X-ray fluorescence spectrum measured from the $L a N / B N$ multilayer. 


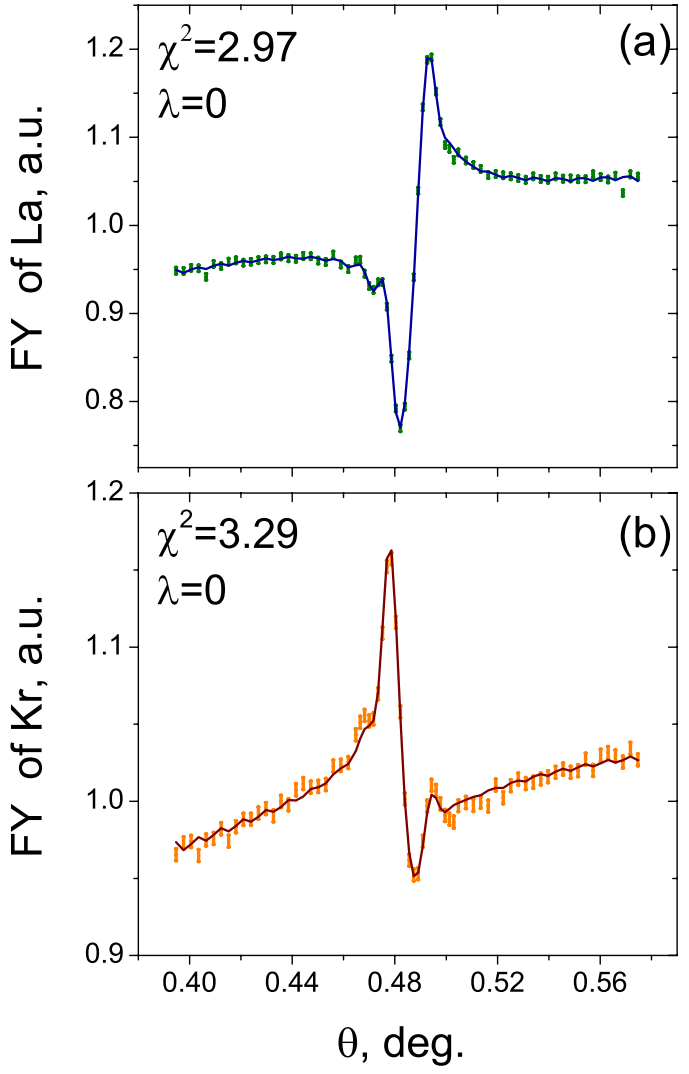

FIG. 4. Experimental (symbols) and simulated (solid lines) X-ray fluorescence yields (FY) for $L a$ (a) and for $K r$ (b) in the region of the first Bragg peak region.

obtained $\chi^{2}$ that need to be compared with the values obtained for a non regularized solution.

To resolve the ill-posed problem, limitations to atomic function were introduced according to Eq. (10). For smoothening of the atomic distribution function, the coefficient $\lambda=10^{-8}$ was used for both element distributions. This value for $\lambda$ was selected to provide the best fit of the measured to calculated XSW data. The fit quality for smoothed atomic distributions is just slightly worse than for not smoothed: $\chi^{2}=6.5$ for $L a$ and $\chi^{2}=4.4$ for $K r$. The resulting atomic distribution functions are presented in Figs. 5(c) and 5(d).

Note that the smooth atomic distribution is still physically impossible because of the locally negative density values for both materials. The smoothening of layers can force the ADF to have negative atomic concentrations to enable artificially smoothed transition between the regions with and without atoms where there are natural splits in ADF. The layers with negative atomic concentrations should then be cancelled by sequential "removal" of the individual equations that correspond to the sub-layers with the largest negative concentrations, followed by searching for a new solution of a lower-rank system of equations. After the sequential removal of negative probability densities and solving of the reduced system of equations (10), the final ADFs were found and are shown in Figs. 5(e) and 5(f). The fit goodness of the final fit $\left(\chi^{2}=4.6\right.$ for $L a$ and $\chi^{2}=3.1$ for $\left.K r\right)$ is actually better than that for the initial smoothed ADF that allowed negative probability densities because of the reduced degrees of freedom during the calculations of the $\chi^{2}$ function.
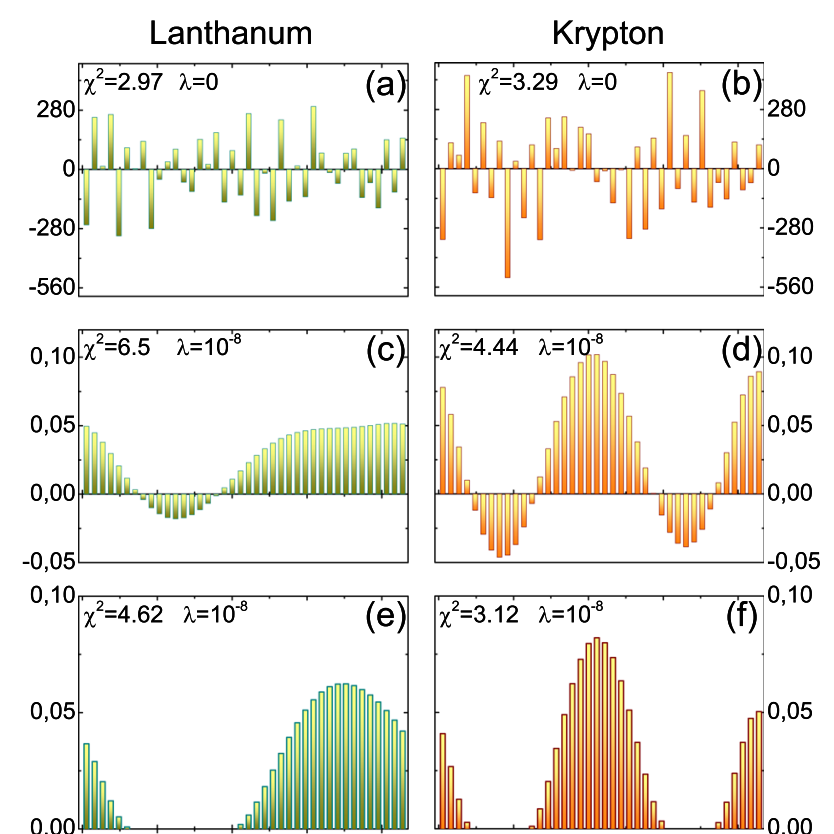

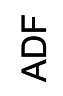
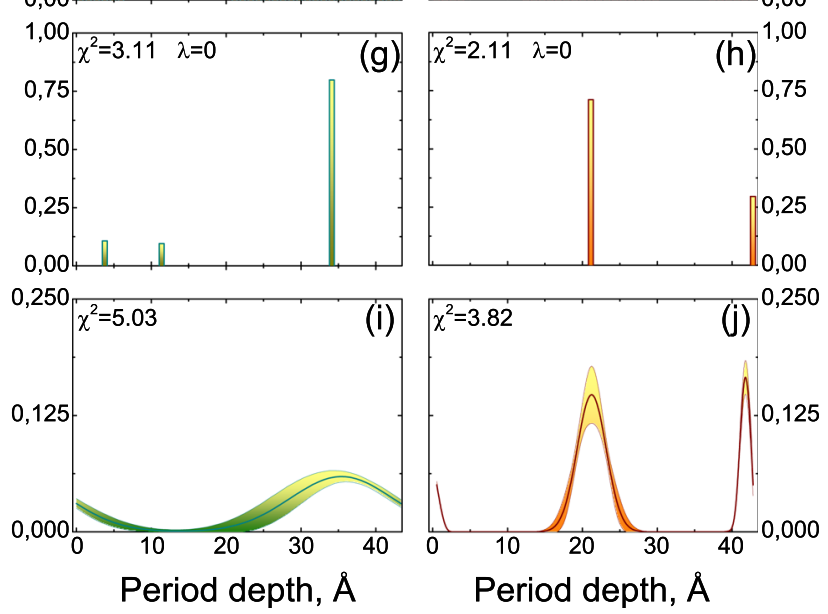

FIG. 5. Reconstructed atomic distribution functions for $L a$ and $K r$ using no function limitations ( $a$ and b), function smoothening (c and d), and smoothening with iterative removal of sublayers with negative values (e and $f$ ). Also shown are non-smoothened functions with iterative removal of sublayers with negative values ( $\mathrm{g}$ and $\mathrm{h}$ ) and functions obtained using Gaussian atomic distributions (i and $\mathrm{j}$ ).

To determine the accuracy and stability of the reconstructed ADFs, additional analysis was performed. Unfortunately, the suggested approach (Eq. (10)) does not allow evaluation of errors in the reconstructed ADFs because the shape of the reconstructed ADF is dependent on the smoothening parameter $\lambda$ which influences errors. To estimate errors and the stability of the determination of ADF, the XSW data can be fitted using a Gaussian shape of the atomic concentration distribution. Errors can be derived from this fit. The fit requires the input of initial parameters for the Gaussian model where the exact positions of a distribution center are the most important model parameters. These positions can be found by the iterative removal of sublayers with negative values in atomic concentrations obtained from the solution of the non regularized system of linear equations (6).

Starting from the non-smoothened (Figs. 5(a) and 5(b)), sub-layers with the highest negative value were iteratively 
removed until no negative values remained, resulting in the ADFs shown in Figs. 5(g) and 5(h). The obtained ADFs correspond to the peak positions of $L a$ distribution profiles. The thus determined ADFs were further resolved by the fit of model based calculations of fluorescence yield to measured XSW data, assuming that concentration ADFs follow a Gaussian distribution form with peak center position and peak width as fit parameters. The center positions of the initial Gauss profiles were obtained from Figs. 5(g) and 5(h). Because of the relative importance of the single peak at $z=35 \AA$, a single Gaussian distribution at this position was used for the analysis of the $L a \mathrm{ADF}$. The result of the reconstruction is presented in Fig. 5(i). The fluorescence yield of $K r$ was simulated with two separate Gaussians located at the positions obtained from Fig. 5(h). Comparing the $L a$ ADFs from Figs. 5(e) and 5(h), we can conclude that all exhibit the same location of $L a$ within the period, within a $1 \AA$ accuracy. The same conclusion can be drawn for $K r$.

\section{DISCUSSION}

The model-independent technique for XSW data analysis presented here expands the series of model independent approaches presented in works Cheng ${ }^{11}$ and $\mathrm{Kohli}^{12}$ and completes the set of model independent approaches for all types of XSW techniques: Bragg-XSW for single crystals, long period XSW for layered structures, and Bragg XSW for periodic multilayer structures.

The benefit of the current approach is that for the reconstruction of ADF by direct solution of Eq. (10), the XSW data can be measured only for one order of Bragg reflection, especially when higher order Bragg peaks show low contrast. The disadvantage is that regularization procedure forces the ADF to be smoothed and inaccurate selection of the smoothening coefficient $l$ may force an artificially smoothened ADF. Taking into account that in Eq. (6) the fluorescence yield is excited by the general EM field shape, the current approach can be extended to the long-period XSW technique. However, the modification of the regularization technique might be required there.

The analysis of errors in the ADFs reconstructed using the XSW technique shows that the position of the maximum in the atomic distribution ADF can be determined with an accuracy of $1 \AA$. The analysis of errors was performed assuming that the EM field does not change with the variation of the atomic profiles, and suggests that the derived error is slightly underestimated. However, if all experimental artifacts connected to the beam spectral and geometrical resolution and goniometric uncertainties are taken into account, the reconstructed positions of atom localization are reliable. We should note that because of the shape of the EM field, an increase of the atomic distribution width will lead to a decrease in the accuracy of the shape of the distribution, reducing the accuracy of the profile width determination. We also note that the XSW technique yields the averaged over all periods profile, and a strong aperiodicity in the sample will therefore be misinterpreted as a blurring of the atomic distribution. The GIXR technique applied here for the EM field reconstruction is sensitive to the periodicity of the

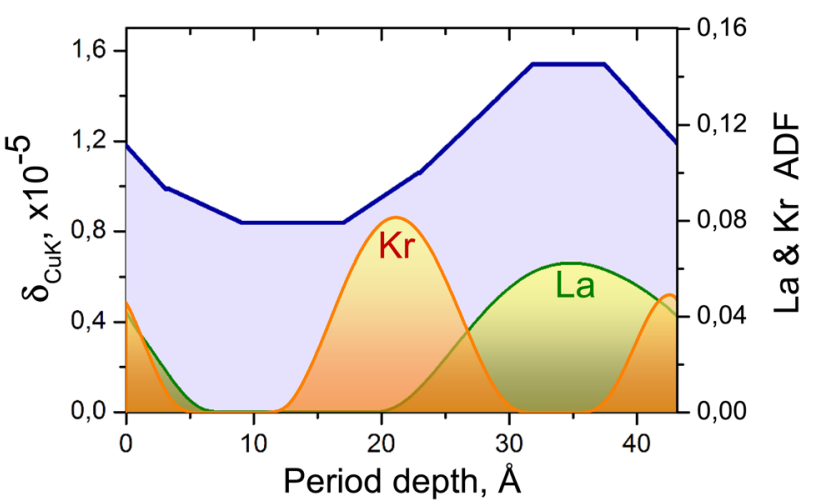

FIG. 6. Comparison of obtained electron density profiles and atomic distribution functions.

multilayer and will allow estimation of period fluctuations before the XSW analysis is performed.

Here, it should be noted that in the presented approach for the reconstruction of the ADF we take into account not only the amplitude and phase of the oscillation of angular fluorescence yield like in classical Fourier analysis of XSW data, but also it's shape, which gives the sensitivity to the shape of the reconstructed ADF. However, because only the first Bragg peak was analyzed in the analysis presented here, fine details of the reconstructed ADF cannot be resolved. The reconstructed ADF can be considered as the probability density for the positions of atoms inside the multilayer period. For a more detailed reconstruction of the atomic profile, the fluorescence yields exited by higher orders of diffraction should be added to the analysis.

Figure 6 shows a comparison between the EDP obtained from GIXR and the ADF obtained from the XSW analysis. As discussed in the previous section, the atomic distribution is presented in terms of a normalized probability density per element. In absolute value, the amount of $K r$ is approximately one order of magnitude less than that of $L a$.

The unique result of the XSW analysis is a non destructive analysis of the impurity distribution. A small residue of the sputtering gas $K r$ could be detected and appears localized in the interface regions. Apparently, $K r$ ions from the magnetron plasma (with energies $300 \mathrm{eV}$ during $L a$ sputtering and $600 \mathrm{eV}$ during $B_{4} C$ sputtering) are capable to penetrate through the already deposited layers and be trapped in the interfaces. The result is also important as $K r$ has a high absorption for the $6.7 \mathrm{~nm}$ wavelength and its presence will reduce the reflectance in the envisioned application.

\section{CONCLUSIONS}

As demonstrated in this paper, the analysis of X-ray standing wave data from periodic multilayer structures based on the solution of linear equations describing the fluorescence yield, allows a fast and model independent reconstruction of atomic distribution function. The approach was applied to Bragg-XSW data from a $L a N / B N$ multilayer structure. The obtained atomic distribution function of the $L a$ atoms in the structure showed an absence of $L a$ 
atoms in the $B N$ layer. Additionally, the contamination of the multilayer by $K r$ atoms, trapped during the magnetron deposition process, was revealed. It was found that these $\mathrm{Kr}$ atoms are distributed inside the interface regions. The sensitivity of the XSW technique to such atoms remains high, even though their presence does not change the electron density profile.

\section{ACKNOWLEDGMENTS}

Part of this work was carried out within the project "Multilayer Optics for Lithography Beyond the Extreme Ultraviolet Wavelength Range" with support of the Dutch Technology Foundation (STW) in the frame of the Thin Film Nanomanufacturing programme. This work is also part of the research programme "Controlling photon and plasma induced processes at EUV optical surfaces (CP3E)" of the "Stichting voor Fundamenteel Onderzoek der Materie (FOM)" with financial support from the "Nederlandse Organisatie voor Wetenschappelijk Onderzoek (NWO)", Carl Zeiss SMT, ASML, and the AgentschapNL through the EXEPT program.

A part of this work was supported by a grant for Russian young scientists and leading scientific schools NSH 5837.2012.2 "Physics of coherent interaction of X-ray and synchrotron radiation with matter, the development of highresolution phase-sensitive X-ray techniques for structural diagnostics of crystalline and nanomaterials" and Grant of
Russian Foundation for Basic Research No. 12-02-12045ofi-m.

${ }^{1}$ A. M. Afanasev and V. G. Kohn, Zh. Eksp. Teor. Fiz. 74, 300 (1978).

${ }^{2}$ M. V. Kovalchuk and V. G. Kohn, Usp. Fiz. Nauk 149, 69 (1986).

${ }^{3} \mathrm{~J}$. Zegenhagen and A. Kazimirov, The X-ray Standing Wave Technique: Principles and Applications: World Scientific Publishing Company Incorporated (World Scientific Publishing Co., Pte., Ltd, 2013).

${ }^{4}$ S. I. Zheludeva et al., Rev. Sci. Instrum. 63(1), 1519 (1992).

${ }^{5}$ M. K. Tiwari, K. J. S. Sawhney, and G. S. Lodha, Surf. Interface Anal. 42(2), 110 (2010).

${ }^{6}$ S. K. Ghose and B. N. Dev, Phys. Rev. B 63, 245409 (2001).

${ }^{7}$ S. I. Zheludeva et al., Thin Solid Films 259, 131 (1995).

${ }^{8}$ M. J. Bedzyk et al., Science 241(4874), 1788 (1988).

${ }^{9}$ A. Gupta et al., Phys. Rev. B 72(7), 075436 (2005).

${ }^{10} \mathrm{~V}$. V. Roddatis et al., J. Mater. Res. 28(11), 1432 (2013).

${ }^{11}$ L. Cheng et al., Phys. Rev. Lett. 90(25), 255503 (2003).

${ }^{12}$ V. Kohli, M. J. Bedzyk, and P. Fenter, Phys. Rev. B 81(5), 054112 (2010).

${ }^{13}$ A. N. Tikhonov and V. Y. Arsenin, Solutions of Ill-Posed Problems (Winston, 1977).

${ }^{14}$ S. N. Yakunin, E. M. Pashaev, A. A. Zaitsev, A. G. Sutyrin and V. G. Mokerov, Proc. SPIE 5401, 573 (2004).

${ }^{15}$ I. A. Makhotkin et al., J. Micro/Nanolithogr., MEMS, MOEMS 11(4), 040501 (2012).

${ }^{16}$ Yu. Platonov et al., Proc. SPIE 8076, EUV and X-Ray Optics: Synergy between Laboratory and Space II, 80760N (4 May 2011).

${ }^{17}$ T. Tsarfati et al., Thin Solid Films 518, 7249 (2010).

${ }^{18}$ A. M. Afanas'ev and M. A. Chuev, J. Theor. Exp. Phys. 80(3), 560 (1995).

${ }^{19}$ T. Hohage, K. Giewekemeyer and T. Salditt, Phys. Rev. E 77(5), 051604 (2008).

${ }^{20}$ F. Abeles, J. Phys. Radium 11, 307 (1950).

${ }^{21}$ M. Born and E. Wolf, Principles of Optics (Cambridge University Press, 1999).

${ }^{22}$ E. Louis et al., Prog. Surf. Sci. 86(11), 255 (2011). 